\title{
Entangled-photons generation with quantum dots
}

\author{
Yuan $\mathrm{Li}^{1}$, Fei Ding ${ }^{* 1,2}$ and Oliver G. Schmidt ${ }^{1}$ \\ 1 Institute for Integrative Nanosciences, IFW Dresden, Helmholtzstraße 20, 01069 Dresden, Germany. \\ 2 Institute for Solid State Physics, Leibniz University of Hannover, Appelstraße 2, 30167 Hannover, Germany.
}

\begin{abstract}
Entanglement between particles is a crucial resource in quantum information processing, an important example of which is the exploitation of entangled photons in quantum communication protocols. Among the different available sources of entangled photons, semiconductor quantum dots (QDs) excel owing to their deterministic emission properties, potential for electrical injections, and direct compatibility with semiconductor manufacturing techniques. Despite the great promises, QD-based sources are far from being ideal. In particular, such sources present several critical issues, which require the overcoming of challenges pertaining to spectral tunability, entanglement fidelity, photon indistinguishability and brightness. In this article, we will discuss the potential solutions to these problems and review the recent progress in the field.
\end{abstract}

\section{Key words: Quantum information, Entangled-photon source, Quantum dots}

PACS: 03.67.Hk, 03.67.Bg, 73.21.La

\section{Introduction}

In 1935, Einstein, Podolsky and Rosen presented a thought experiment to describe the peculiarity of the quantum entanglement. ${ }^{[1]}$ In a many-body entanglement system, the quantum state of each constituting particle cannot be described independently, even when the particles are separated by large distances. The exploitation of the entanglement in quantum information processing (QIP), e.g., quantum computing, ${ }^{[2,3]}$ quantum cryptography ${ }^{[4,5]}$ and quantum teleportation, ${ }^{[6,7]}$ has attracted significant attentions in the last decade. In this regard, it is crucial to develop high-quality sources for entangled quantum bits (qubits). At present, the generation of entangled qubits has been realized in various systems, such as atomic ions, ${ }^{[8,9]}$ superconductor circuits, ${ }^{[10]}$ electrons, ${ }^{[11,12]}$ diamonds, ${ }^{[13]}$ and photons. ${ }^{[14,15]}$ In this article, we focus on the recent developments of polarization-entangled photons generated by semiconductor quantum dots (QDs).

\section{Quantum bits}

\subsection{Concept}

In analogy to the classical information science, which uses the binary bits 0 and 1 to store information, the basic entity of quantum information is the qubit. The basic states to form an orthogonal basis of the qubit space are called computational bases. Considering a two-state system, for example, if the computational bases are $|0\rangle$ and $|1\rangle$, one possible pure qubit can be described as $|\psi\rangle=a|0\rangle+b|1\rangle$, where $a$ and $b$ are complex coefficients and they respect the normalization condition $|a|^{2}+|b|^{2}=1$. In the classical information theory, a bit must be either 0 or 1 , whereas in quantum theory, a qubit can be in both the $|0\rangle$ and $|1\rangle$ state at the same time. Such condition represents the coherent superposition of $|0\rangle$ and $|1\rangle$. In the attempt of measuring the state of a qubit, one would obtain either $|0\rangle$ or $|1\rangle$, with a probability of ||$^{2}$ or $\mid b^{2}$, respectively. The coherent superposition and the probabilistic nature are the most distinct characters of qubits compared to classical binary bits. To date, qubits have been realized in several real physical systems. Figure 1 reports a list of some physical realizations of qubits, where the bases have been chosen by convention. Despite the great research efforts, most of these systems require rigorous operating conditions, such as ultra-low temperatures and almost adiabatic environments. At the present stage, photons are ideal candidates for many QIP tasks (e.g., quantum communication), given their weak interaction with the environment, the long coherent time, the fact that they travel at the speed of light and allow a multiple degree-offreedom encoding. 


\begin{tabular}{|c|c|c|c|}
\hline Quantum system & Property & $|0\rangle$ & $|1\rangle$ \\
\hline \multirow{2}{*}{ Photon } & Polarization & Horizontal & Vertical \\
\cline { 2 - 4 } & Arrival time & Early & Late \\
\hline Electron & Spin & Up & Down \\
\hline Nucleus & Nuclear spin & Up & Down \\
\hline Two-level atom & State & Ground state & Excited state \\
\hline Josephson junction & Charge & N Cooper pairs & $\mathrm{N}+1$ Cooper pairs \\
\hline
\end{tabular}

Figure 1. Common physical realizations of qubits. ${ }^{[16]}$ The figure refers to Ref. 16.

\subsection{Photonic qubits}

The photon, whose modern concept was developed by Albert Einstein, is a fundamental particle exhibiting the wave-particle duality. In other words, photons are self-oscillating short electromagnetic waves and particles with spin 1 at the same time. Photons can be used as flying qubits in QIP, due to their unique properties mentioned above. In particular, a single photon possesses many degrees of freedom (e.g., spin angular momentum, orbital angular momentum, arrival time, frequency, and so on), which can be used to encode a coherent superposition of states. Figure 2 shows the Bloch sphere representations of three commonly used qubit encoding schemes.

(a) Polarization qubit

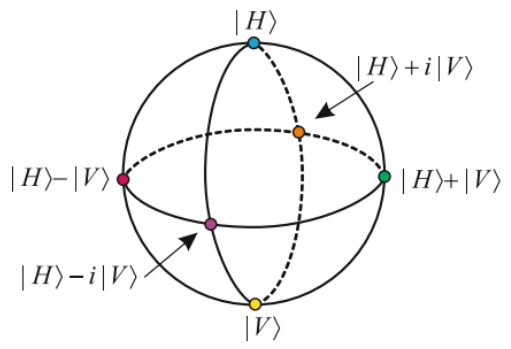

(b) OAM qubit

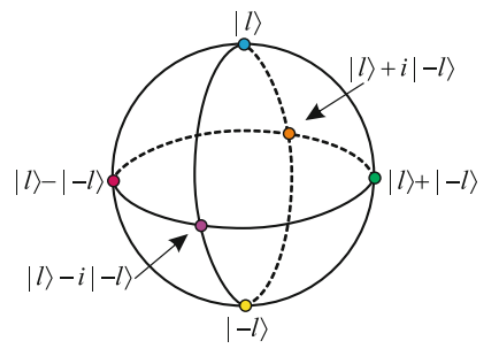

(c) Time-bin qubit

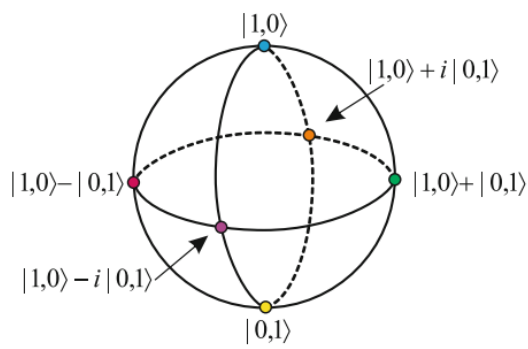

Figure 2. Bloch sphere representations of (a) polarization qubit, (b) orbital angular momentum qubit and (c) time-bin qubit. ${ }^{[17]}$ The figure is adapted from Ref. 17.

\section{Polarization qubits}

If we describe the photon as an electromagnetic wave, its polarization represents the oscillation direction of the electric field. In this viewpoint, the circular polarization is the spin angular momentum of the photon, with the left-handed and right-handed circular polarizations corresponding to $t h$ and $-\mathrm{h}$ spin state, respectively. As shown in Fig. 2(a), each pair of opposite vertices on the Bloch sphere represents one pair of orthonormal bases. $|H\rangle$ and $|V\rangle$ denote the horizontal and vertical polarizations, respectively. Therefore, $|H\rangle \pm|V\rangle$ and $|H\rangle \pm i|V\rangle$ denote the diagonal and circular polarization bases, respectively. The pure states are on the Bloch sphere, whereas the mixed states are in the interior of the Bloch sphere. The polarization encoding of photon qubits is one of the most mature techniques used in modern quantum optics.

\section{Orbital angular momentum qubits}

The orbital angular momentum (OAM) of photons, which represents the spatial distribution of the electromagnetic field, is associated with the helical or twisted phase front. The schematic is shown in Fig. 3. ${ }^{[18]}$ Unlike the spin angular momentum of photons, which has only two computational bases, the OAM can assume any arbitrary integer value, and thus, offer a unique possibility for a high-dimensional encoding. One pair of orthonormal bases is shown in Fig. 2(b), where $l$ denotes the angular quantum number. Ever since the first proposal, ${ }^{[19]}$ significant efforts have been made to use the OAM encoding in QIP. ${ }^{[18,20]}$ In recent years, one of the most striking results is the realization of a spin-OAM hyper-entanglement for single photons. ${ }^{[21,22]}$ 


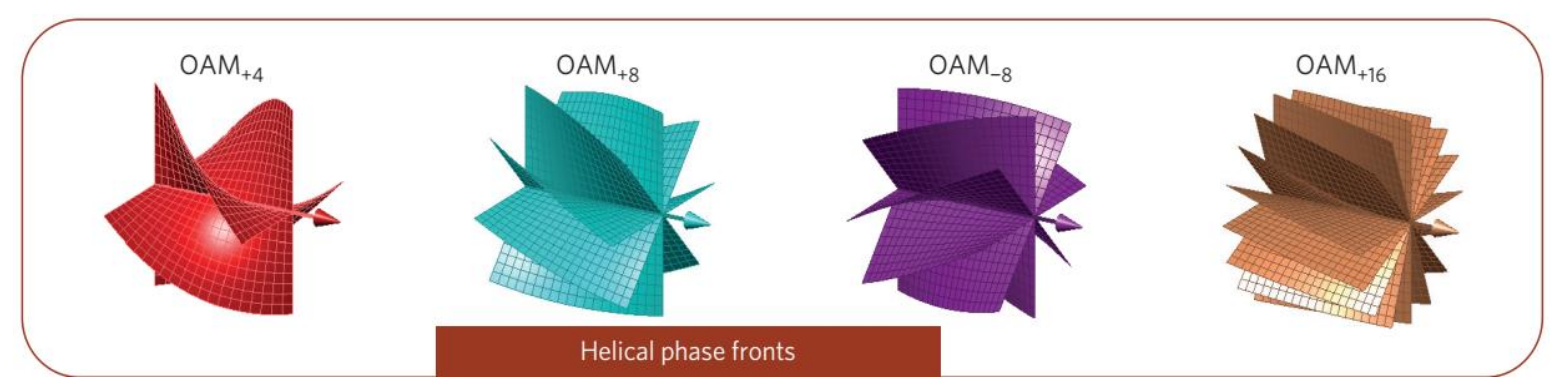

Figure 3. Helical phase fronts of OAMs with different indexes. ${ }^{[18]}$ The figure is adapted from Ref. 18.

\section{Time-bin qubits}

The time-bin encoding is based on the arrival time of a single photon. A detailed experimental configuration was firstly proposed by Brendel. ${ }^{[23]}$ A photon is sent into a two-path setup, then the quantum state of the photon can be described by $|1,0\rangle$ or $|1,0\rangle$ (see also Fig. 2(c)). Here, to ensure the unambiguous distinguishability of the two paths, the difference between the lengths of the two paths should be larger than the coherent length of the photon. The key point is to use a variable coupler and an unbalanced Mach-Zehnder interferometer (phase shifter) in one path to prepare and measure the qubits. ${ }^{[2]}$ The time-bin encoding greatly simplifies the realizations of twophoton quantum cryptography, quantum teleportation, dense coding, entanglement swapping, and GHZ-states sources. ${ }^{[23]}$ Furthermore, due to the robustness against the polarization mode dispersion, time-bin qubits are suitable for applications based on fiber optics. However, the environment insensitivity of time-bin qubits makes it difficult to perform quantum interferences. Building up an effective interference scheme is the goal to achieve.

\section{Quantum entanglement}

\subsection{Concept}

The quantum entanglement describes a nonlocal property of quantum correlations between multiple systems. It can be described mathematically in an elegant way. Here, we refer to a two-body system for example. Let us consider two physical subsystems $\mathrm{A}$ and $\mathrm{B}$, whose respective Hilbert spaces are $\mathrm{H}_{\mathrm{A}}$ and $\mathrm{H}_{\mathrm{B}}$, and $|i\rangle_{A}$ and $|j\rangle_{B}$ are the bases of $\mathrm{H}_{\mathrm{A}}$ and $\mathrm{H}_{\mathrm{B}}$, respectively. The quantum state of the composite system $\mathrm{AB}$ can be described as

$$
|\psi\rangle_{A B} \equiv \sum_{i, j} c_{i j}|i\rangle_{A}|j\rangle_{B}
$$

where $c_{i j}$ indicates the complex coefficients of the composite state. The indexes $\mathrm{i}$ and $\mathrm{j}$ run over the dimensions of $\mathrm{H}_{\mathrm{A}}$ and $\mathrm{H}_{\mathrm{B}}$, respectively. Furthermore, if $|\psi\rangle_{A B}$ can be described by Eq. (2),

$$
|\psi\rangle_{A B}=\sum_{i} a_{i}|i\rangle_{A} \otimes \sum_{j} b_{j}|j\rangle_{B}=|\varphi\rangle_{A} \otimes|\varphi\rangle_{B}
$$

it will be a separable state. Otherwise, if $|\psi\rangle_{A B}$ can only be described by Eq. (1), and not by Eq. (2), it will be an inseparable state, that is an entangled state. The mathematical definition of the quantum entanglement is straightforward, whereas the physical origin of the entanglement is still under debate.

\subsection{Polarization-entangled photon pairs}

\section{Description}

In the following we mainly focus on polarization-entangled photon pairs. The electric vector of an electromagnetic wave can be described by any orthogonal polarization basis, such as horizontal $(|H\rangle)$ and vertical $(|V\rangle)$ bases; left circular $(|L\rangle)$ and right circular $(|R\rangle)$ bases; diagonal $(|D\rangle)$ and antidiagonal $(|A\rangle)$ bases. Then, the polarization entangled Bell states can be described as follows:

$$
\begin{aligned}
& \left|\Phi^{ \pm}\right\rangle=\frac{1}{\sqrt{2}}(|H H\rangle \pm|V V\rangle) \\
& \left|\Psi^{ \pm}\right\rangle=\frac{1}{\sqrt{2}}(|H V\rangle \pm|V H\rangle)
\end{aligned} .
$$


One can use coordinate system transformations, such as those in Eq. (4), to express the Bell state in the other two polarization bases.

$$
\begin{aligned}
& |H\rangle=\frac{1}{\sqrt{2}}(|L\rangle+|R\rangle)=\frac{1}{\sqrt{2}}(|D\rangle+|A\rangle) . \\
& |V\rangle=\frac{i}{\sqrt{2}}(|L\rangle-|R\rangle)=\frac{1}{\sqrt{2}}(|D\rangle-|A\rangle)
\end{aligned}
$$

The so-called Bell states in Eq. (3) are the maximally entangled states. In practice, the inevitable quantum decoherence induced by the environment will degrade the fidelity of the entanglement.

\section{Generation processes}

\section{(a) Atomic cascade}

In the early years, polarization-entangled photon pairs were generated from the cascade radiations of single calcium $(\mathrm{Ca})$ atoms. ${ }^{[25,26]}$ The schematic diagram is shown in Fig. 4.

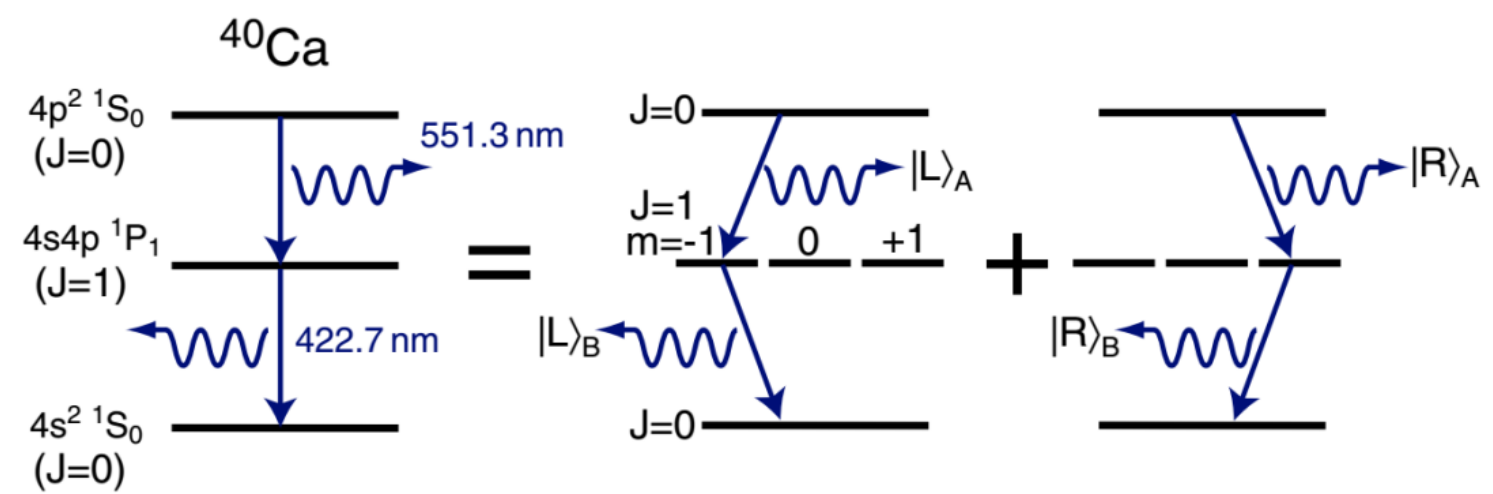

Figure 4. Two photons cascade radiation in a Ca atom. ${ }^{[27]}$ Figures are adapted from Ref. 27.

By using the two-photon pumping technique, one electron is excited from the ground energy level $4 s^{2}{ }^{1} S_{0}$ to the excited level $4 \mathrm{p}^{2}{ }^{1} \mathrm{~S}_{0}$. Then, the electron jumps down to the ground state via the intermediate level $4 \mathrm{~s} 4 \mathrm{p}{ }^{1} \mathrm{P}_{1}$. Due to the triple degeneration of the intermediate state, two entangled photons A and B, with wavelengths of 551.3 $\mathrm{nm}$ and $422.7 \mathrm{~nm}$, respectively, are emitted during the recombination process. The polarization state of the pair $\mathrm{AB}$ of the entangled photons can be written in the form

$$
|\psi\rangle_{A B}=\frac{1}{\sqrt{2}}(|L L\rangle+|R R\rangle)
$$

Aspect et al. experimentally demonstrated that the generated photon pairs are in one of the maximally entangled Bell states. However, the single-atom based source has several natural drawbacks: it is difficult to handle, and the photons are emitted in random space angles; therefore, its application in QIP is limited.

\section{(b) Spontaneous parametric down-conversion}

Spontaneous parametric down-conversion (SPDC), which was observed in the 1960s, describes a nonlinear optical effect in crystals, such as beta-barium borate (BBO) and potassium dihydrogen phosphate (KDP). More specifically, when a pump light is irradiated on a nonlinear crystal, a small portion of photons will interact with the crystal itself to generate down converted photons. In this process, the pump photon will generate a signal photon and an idler photon, and the three photons must fulfill the energy and momentum conservations, as well as the phase matching condition. Signal and idler photons are entangled in their polarizations. As shown in Fig. 5, there are two types of SPDC setups that can generate polarization-entangled photon pairs. 
(a)

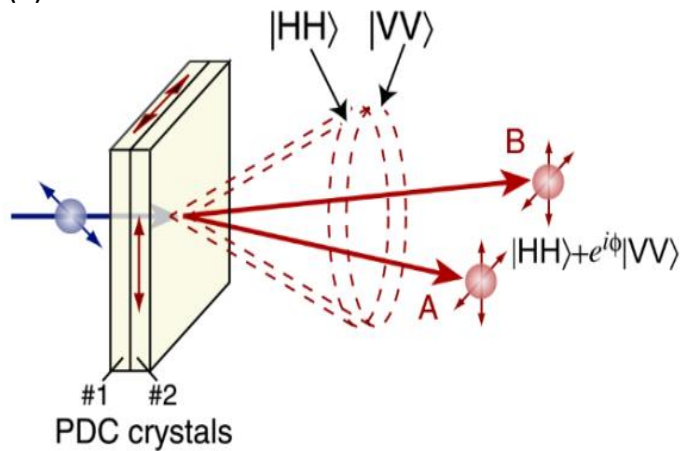

(b)

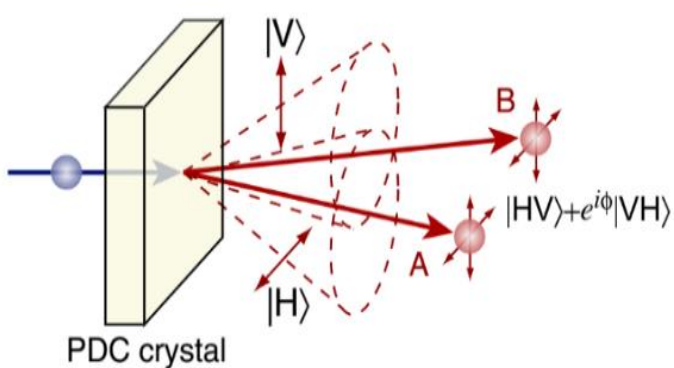

Figure 5. Type-I (a) and Type-II (b) spontaneous parametric down-conversion. ${ }^{[27]}$ Figures are adapted from Ref. 27.

In type-I SPDC, the two output photons have the same polarization direction, whereas in type-II SPDC, the two output photons have polarization directions perpendicular to each other. The corresponding entangled states are also shown in Fig. 5. To date, SPDC is the main workhorse for the generation of entangled photon pairs and single photons in QIP. However, the generated photons are characterized by Poissonian statistics, i.e., they are created at random time. Besides, the conversion efficiency is only on the order of 1 pair output per $10^{12}$ incoming photons. ${ }^{[28]}$ The randomness and low efficiency of SPDC limit its application in complex QIP.

\section{(c) Semiconductor quantum dots}

The III-V group semiconductor QDs, particularly QDs based on indium arsenide (InAs) and gallium arsenide (GaAs), possess the type-I energy-band alignment and can efficiently confine both electrons and holes in nanometer dimensions. As a result, the exciton effect is dominating in emission. When the QDs are symmetric enough, they can be the leading candidates for a deterministic generation of polarization-entangled photons. We will discuss the influence of symmetry on entanglement properties of QDs' emission later, for the moment we only consider the QDs having a $\mathrm{D}_{2 \mathrm{~d}}$ symmetry. Once a $\mathrm{D}_{2 \mathrm{~d}} \mathrm{QD}$ is in the biexciton state, the radiative decay process can give out two photons consecutively via one of the two energy-degenerated intermediate exciton states, as shown in Fig. $6 .{ }^{[29]}$ The two photons are maximally polarization-entangled. The entangled two-photon state can be described in different bases, as it follows:

$$
|\psi\rangle_{A B}=\frac{1}{\sqrt{2}}(|L R\rangle+|R L\rangle) \equiv \frac{1}{\sqrt{2}}(|H H\rangle+|V V\rangle) \equiv \frac{1}{\sqrt{2}}(|D D\rangle+|A A\rangle)
$$

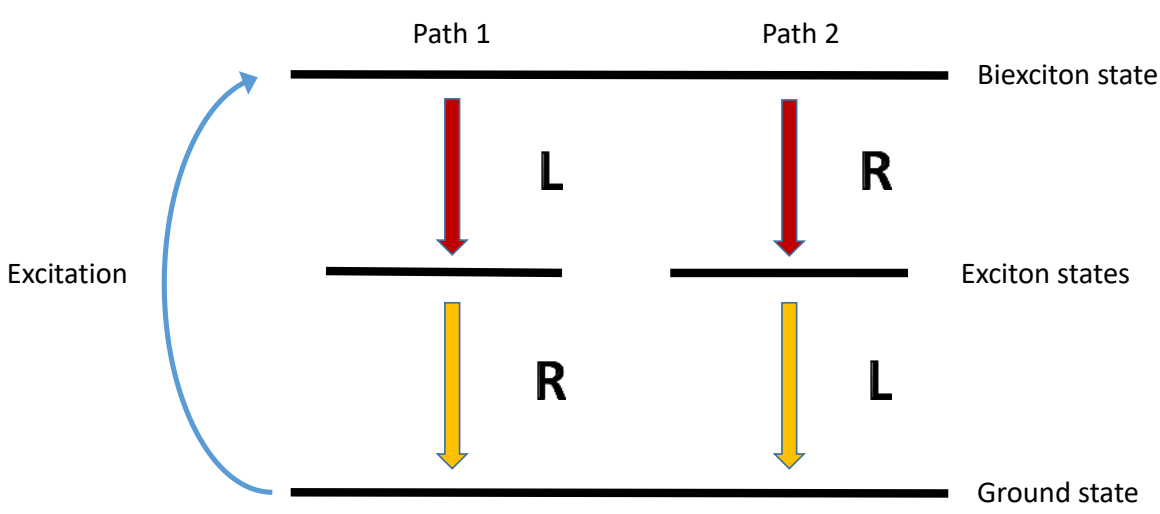

Figure 6. Illustration of the radiative decay process for a $\mathrm{D}_{2 \mathrm{~d}}$ semiconductor $\mathrm{QD}$. L and $\mathrm{R}$ denote left and right circularly polarized photons respectively.

A major advantage of QDs sources is the deterministic generation of one pair of entangled photons per excitation pulse, which overcomes the drawback of SPDC. Such deterministic generation makes QDs to be promising candidates for quantum emitters. This scheme was first proposed by Benson et al., ${ }^{[30]}$ and significant progresses have been made since. Later, we will show that the symmetry of QDs is the limiting element in the degree of entanglement. 


\section{Evaluation of entanglement}

\section{(a) CHSH inequality}

In 1964, John Stewart Bell proposed a mathematic formulation (the famous Bell inequality) to test the local hidden variable theories. Five years later, John Clauser, Michael Horne, Abner Shimony, and Richard Holt generalized the Bell inequality to CHSH inequality, and proposed a practical experiment to test the EPR paradox. Since then, many endeavors have been made to realize loophole-free Bell inequality tests. ${ }^{[25,31-37]}$ According to the Bell theorem, the key points to verify the entanglement of two particles are the simultaneous measure of the physical properties of the two particles and the analysis of their correlations. The Hanbury Brown and Twiss (HBT) setup is usually employed in these types of experiments, as shown in Fig. 7.

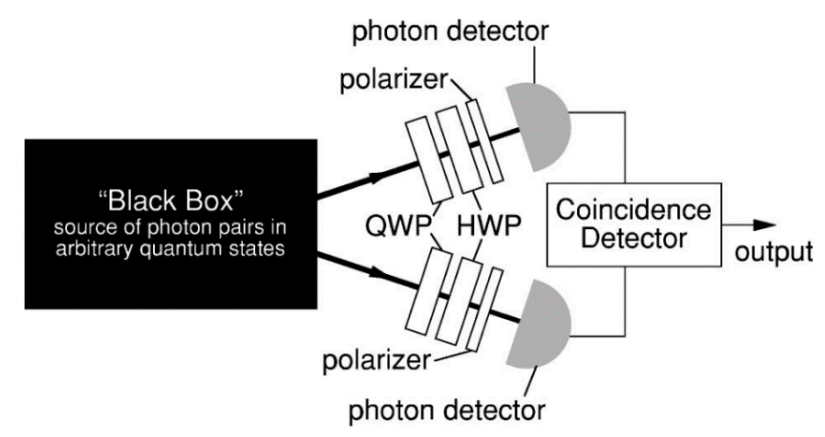

Figure 7. Two-channel photon correlation detection setup. ${ }^{[38]}$ QWP and HWP denote quarter wave plate and half wave plate, respectively. The figure is adapted from Ref. 38.

The black box stands for a source of generation of photon pairs. In the experiment, the generated two photons are separated into two different channels by a non-polarizing beam splitter. The quarter wave plate, half wave plate and polarizer are used to project the photon onto different polarization bases in each channel. The avalanche photodetector and time-correlated single-photon counting system are used to analyze the correlations between the two photons.

After obtaining the correlation data, there are two methods to test the degree of polarization entanglement of the two-photon state. The first method consists in the direct examination of the CHSH inequality, whereas the second one is the quantum state tomography. The $\mathrm{CHSH}$ inequality can be written as ${ }^{[27,39]}$

$$
\begin{aligned}
& S=\left|E(\mathrm{a}, \mathrm{b})-E\left(\mathrm{a}, \mathrm{b}^{\prime}\right)+E\left(\mathrm{a}^{\prime}, \mathrm{b}\right)+E\left(\mathrm{a}^{\prime}, \mathrm{b}^{\prime}\right)\right| \leq 2 \\
& E(\mathrm{a}, \mathrm{b})=\frac{C(\mathrm{a}, \mathrm{b})+C(\overline{\mathrm{a}}, \overline{\mathrm{b}})-C(\overline{\mathrm{a}}, \mathrm{b})-C(\mathrm{a}, \overline{\mathrm{b}})}{C(\mathrm{a}, \mathrm{b})+C(\overline{\mathrm{a}}, \overline{\mathrm{b}})+C(\overline{\mathrm{a}}, \mathrm{b})+C(\mathrm{a}, \overline{\mathrm{b}})} . \\
& \overline{\mathrm{a}}(\overline{\mathrm{b}})=a(b)+90^{\circ}
\end{aligned}
$$

where $C(\mathrm{a}, \mathrm{b})$ is the coincidence count when the first and second channels are at " $\mathrm{a}$ " and "b" polarization angles,

respectively. The notations $a^{\prime}$ and $b^{\prime}$ denote other two polarization angles for the first and second channels, respectively. If the result is $S>2$, it means that the photon pair is polarization-entangled. For the ideal Bell states described by Eq. (3), one would get $S=2 \sqrt{2}$.

\section{(b) Quantum tomography}

Another way to evaluate the entanglement is to reconstruct the density matrix of the studied quantum system. For a two-photon AB system, the polarization state $|\Psi\rangle_{A B}$ can be written as

$$
\begin{aligned}
& |\Psi\rangle_{A B}=m|H H\rangle+n|H V\rangle+p|V H\rangle+q|V V\rangle \\
& \rho_{A B}=|\Psi\rangle_{A B}\left\langle\left.\Psi\right|_{A B}=\left[\begin{array}{c}
m \\
n \\
p \\
q
\end{array}\right]\left[\begin{array}{llll}
m^{*} & n^{*} & p^{*} & q^{*}
\end{array}\right] .\right.
\end{aligned}
$$

According to the quantum tomography theory, ${ }^{[38,40]}$ the density matrix can also be described in terms of experimentally observable quantities: 


$$
\begin{aligned}
& \rho_{A B}=\frac{1}{2^{2}} \sum_{j, k=0}^{3} \frac{S_{j, k}}{S_{0,0}} \sigma_{j} \otimes \sigma_{k} \\
& =\frac{1}{2^{2}} \sum_{j, k=0}^{3}\left\{f_{j, k}\left[\mathrm{~g}_{A \gamma_{1}, \mathrm{~B} \gamma_{2}}^{(2)}(\tau=0)\right]\right\} \times\left(\sigma_{A j} \otimes \sigma_{B k}\right) \\
& \gamma_{1}, \gamma_{2} \in\{H, V, D, A, R, L\}
\end{aligned}
$$

In Eq. (9), $\mathrm{g}_{A \gamma_{1}, \mathrm{~B} \gamma_{2}}^{(2)}$ denotes the second-order correlation function, which is determined by projecting A's and B's polarization states onto $\gamma_{1}$ and $\gamma_{2}$ bases, respectively. The experimental setup to measure the second-order correlation function is illustrated in Fig. 8. The Pauli-like matrixes $\sigma_{j(k)}$ and the function $f_{j, k}$ are described in detail in Ref. 40.

\section{(c) Entanglement fidelity}

In addition to the quantum tomography, the entanglement fidelity is often used as an easily determinable quantity to characterize the degree of an entanglement. In general, the fidelity represents the closeness of one state to another. For example, the entanglement fidelity of a two-photon polarization state to $\left|\Phi^{+}\right\rangle$(see Eq. (3)) can be described as

$$
\begin{aligned}
& \text { Fidelity }=\left\langle\Phi^{+}\left|\rho_{A B}\right| \Phi^{+}\right\rangle \\
& \left|\Phi^{+}\right\rangle=\frac{1}{\sqrt{2}}(|H H\rangle+|V V\rangle)
\end{aligned}
$$

The upper limit of the fidelity of any classical state to a maximally-entangled state is 0.5 . A fidelity larger than 0.5 denotes that the system is in an entangled state. From an experimental point of view, by measuring the second-order correlation function with the HBT setup, one can get the entanglement fidelity to $\left|\Phi^{+}\right\rangle$:

$$
\begin{gathered}
\text { Fidelity }=\frac{1+C_{H ; V}+C_{D ; A}-C_{R ; L}}{4} \\
C_{H(\mathrm{D}, \mathrm{R}) ; \mathrm{V}(\mathrm{A}, \mathrm{L})}=\frac{\mathrm{g}_{\mathrm{H}(\mathrm{D}, \mathrm{R}) ; \mathrm{H}(\mathrm{D}, \mathrm{R})}^{(2)}(0)-\mathrm{g}_{\mathrm{H}(\mathrm{D}, \mathrm{R}) ; \mathrm{V}(\mathrm{A}, \mathrm{L})}^{(2)}(0)}{\mathrm{g}_{\mathrm{H}(\mathrm{D}, \mathrm{R}) \mathrm{H}(\mathrm{D}, \mathrm{R})}^{(2)}(0)+\mathrm{g}_{\mathrm{H}(\mathrm{D}, \mathrm{R}) ; \mathrm{V}(\mathrm{A}, \mathrm{L})}(0)}
\end{gathered}
$$

\section{Semiconductor QD-based polarization-entangled photon sources}

\subsection{Atom-like properties of QD}

Semiconductor QDs are zero-dimensional nanostructures, and their optical and electrical properties can be artificially tailored by shape, size and compositions. Due to the strong quantum confinement, excitons-related effects are dominating in QDs. Such feature makes semiconductor QDs eligible for application opportunities in quantum optics. 


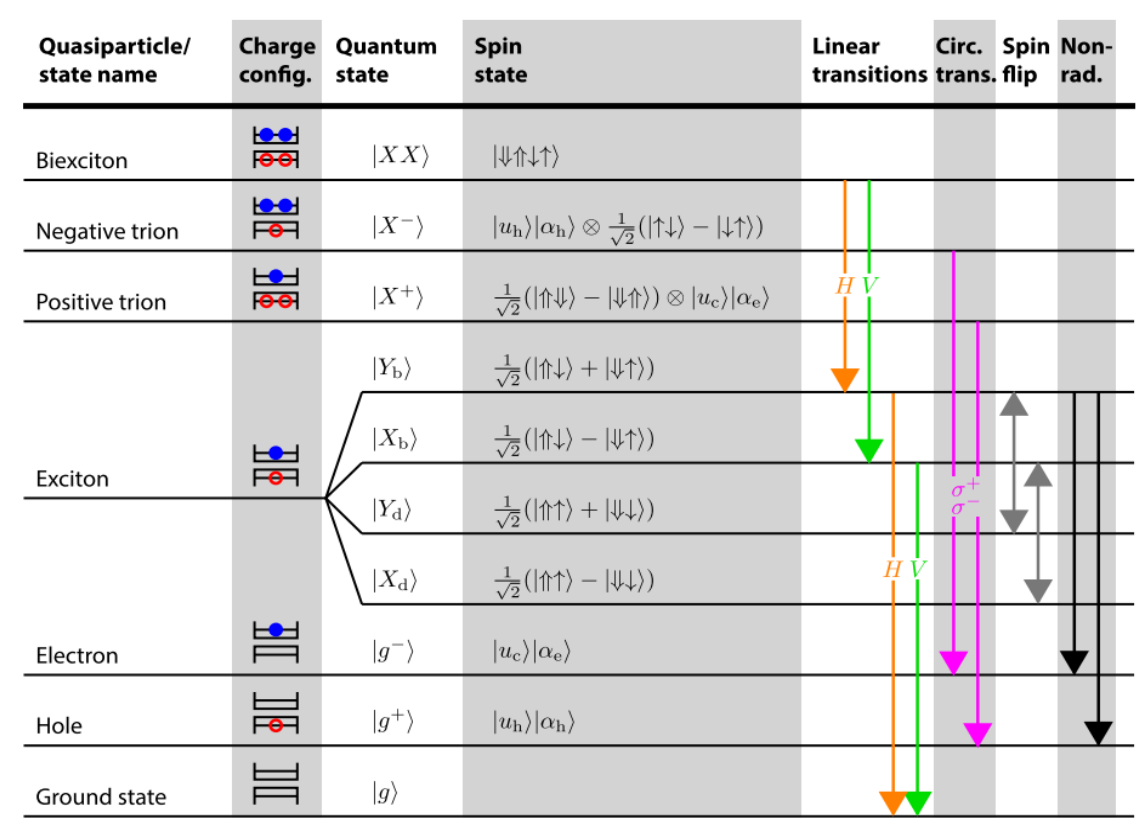

Figure 8. Group of basic exciton states in semiconductor QDs. ${ }^{[41]}$ The double and single arrows in Dirac kets indicate the spin directions of holes and electrons, respectively. $\mathrm{X}, \mathrm{XX}$ and $\mathrm{g}$ denote exciton, biexciton and ground states, respectively. The subscripts $\mathrm{b}$ and $\mathrm{d}$ denote bright and dark excitons, respectively. The figure is adapted from Ref. 41.

The various exciton states in a single III-V group semiconductor QD are shown in Fig. 8. The biexciton-exciton cascade process is used for the generation of polarization-entangled photon pairs, and the entanglement quality is definitely influenced by the QDs. In the next section, we will briefly review the optimization endeavors of QDs for entangled photon emitters.

\subsection{State of art}

As far as the III-V group semiconductor QDs concern, here we mainly focus on the InAs- and GaAs-based ones. In this context, the anisotropy of strain, composition and shape reduces the symmetry of QDs to $C_{2 \mathrm{v}}$ or even lower $C_{1}$. Considering the exchange interaction, there will be an energetic splitting between the two bright excitons, which is called fine-structure splitting (FSS), as shown in Fig. 8. More specifically, when the FSS appears in QDs, the two-photon polarization state generated by the biexciton-exciton cascade process should be described by

$$
|\psi\rangle_{A B}=\frac{1}{\sqrt{2}}\left(\left|H_{A} H_{B}\right\rangle+e^{i \frac{\delta \tau}{\hbar}}\left|V_{A} V_{B}\right\rangle\right) .
$$

where $\delta$ denotes the FSS energy and $\tau$ is the time delay between the emission of biexciton (A) and exciton (B) photons in one cascade process. In this case, the two-photon state periodically recovers to the maximum entanglement Bell state $\left|\Phi^{+}\right\rangle$. Hence, the variation of $\tau$ may destroy the average entanglement fidelity. The time-

gate technique could relieve this problem, ${ }^{[42]}$ but it decreases the source of brightness at the same time. An alternative solution is to suppress the FSS when the FSS is small enough (usually smaller than the lifetime broadened linewidth of exciton). In this case, the time-averaged two-photon state will always be close to $\left|\Phi^{+}\right\rangle$and it will keep a high fidelity. Hence, the FSS tuning is one of the core challenges. Starting from the theoretical part, it is not straightforward to illustrate how the FSS varies with external factors. By adopting an atomistic pseudopotential method, A. Zunger and G. Bester contributed a lot to the theoretical aspect. ${ }^{[3-45]}$ Despite the sophisticated mathematics, Singh et al. calculated the lower bound of FSS in self-assembled QDs under uniaxial stress from the viewpoint of symmetry. ${ }^{[46]}$ As shown in Fig. 9, the QDs excitons belong to various irreducible representations. They claimed that only when the two bright excitons belong to different irreducible representations, the FSS could be eliminated by uniaxial stress. 


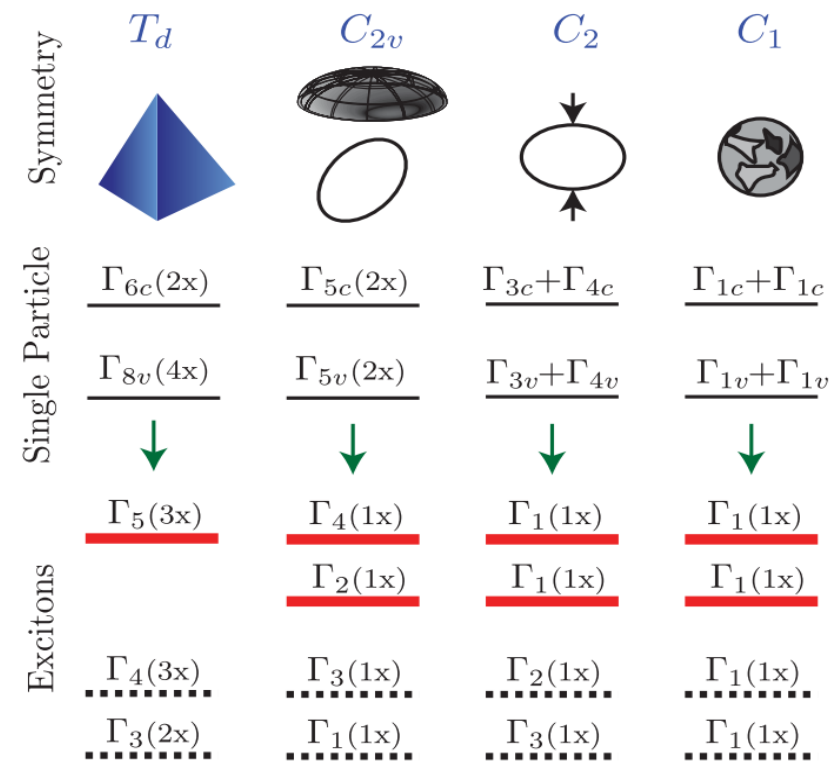

Figure 9. Symmetry analysis for four different point groups. The single-particle levels for the highest occupied and lowest unoccupied states are indicated by solid black lines. The ensuing irreducible representation of bright and dark excitons are indicated by thick red and dashed black lines, respectively. ${ }^{[46]}$ The figure is adapted from Ref. 46.

More systematically, by constructing an effective two-band model based on the two bright excitons, Gong et al. claimed that the lower bound of FSS under uniaxial stress can be predicted by the polarization angle, and that both the FSS under zero stress and the critical stress can be determined by monitoring the changes in the polarization angle of the exciton. ${ }^{[47]}$ The FSS is related to the uniaxial stress according to a parabolic function; therefore, it is possible that the FSS cannot go down to zero under a mere uniaxial stress. This provides a feasible method to select QDs for entangled-photon sources.

Because semiconductor QDs are used as entangled-photon sources, the community has made great efforts to eliminate FSS. An ideal quantum light source, which can be excited either optically or electrically, should possess good properties of brightness, entanglement fidelity, indistinguishability, coherence and on-demand (tunable wavelength and repetition frequency) at the same time. For semiconductor QD-based polarizationentangled photon sources (PEPS), there are three main tasks to be carried out: the manipulation of FSS; the optimization of the excitation condition; and the rational design of optical structures. In recent years, we have witnessed great success in this field.

\section{Growth of highly symmetric QDs}

The FSS mainly originates from the structure asymmetry; therefore its manipulation via the growth control of QDs is straightforward. However, typical InAs QDs grown on GaAs(001) crystal plane using the Stranski-

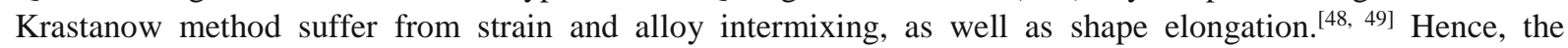
symmetry of this type of QDs is lower than $\mathrm{C}_{2 \mathrm{v}}$. In 2009, two theoretical articles ${ }^{[50,51]}$ predicted that InAs QDs on $\operatorname{GaAs}(111)$ crystal plane would have a symmetry higher than $\mathrm{C}_{3 \mathrm{v}}$, hence a small FSS. The results of the calculation of the wave function symmetry are shown in Figs. 10(a) and 10(b) (see Ref. 50). However, it has been proven that the direct growth of InAs QDs on the GaAs(111) crystal plane is difficult. In 2010, Mohan et al. realized a site-controlled pyramidal QDs grown on tetrahedral recesses patterned (111)B GaAs substrates, which exhibited a $\mathrm{C}_{3 \mathrm{v}}$ symmetry. ${ }^{[52]}$ In 2013, Juska et al. reported on the (111)-grown pyramidal site-controlled InGaAs $1-x N_{x}$ QDs and they demonstrated that the $15 \%$ of their QDs have an entanglement fidelity greater than 0.5. ${ }^{[53]}$ Figures 10(d) and 10(e) show the morphologies of these QDs. 

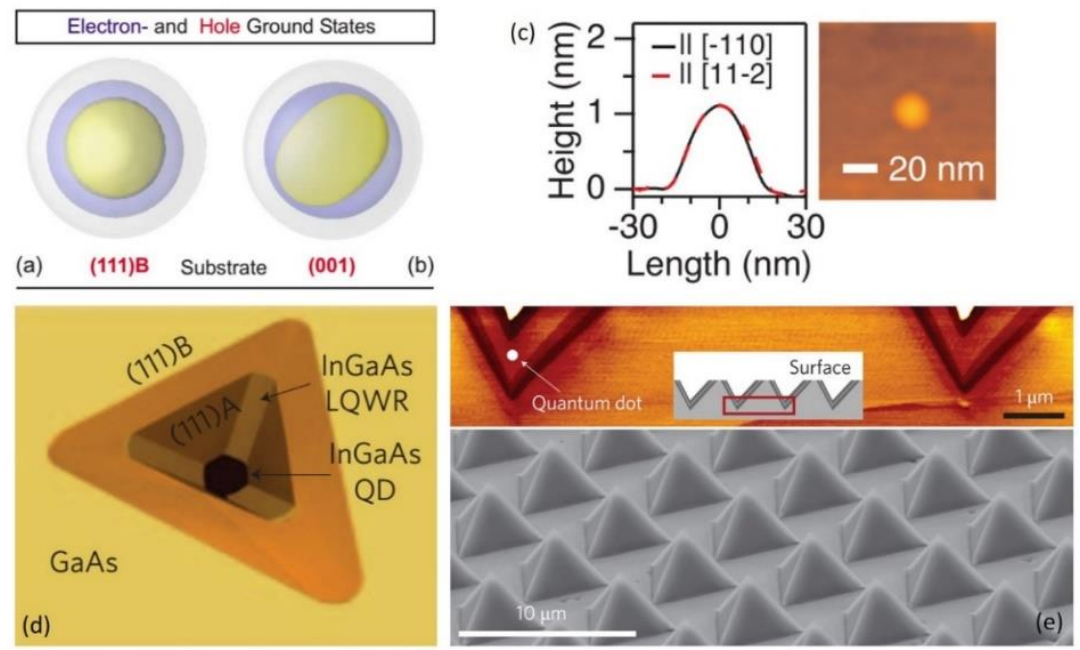

Figure 10. (a) and (b) Orientation of electron (blue) and hole (yellow) wave functions for a lens-shaped QD on two substrates with different orientations. ${ }^{[50]}$ (c) Atomic force microscope analysis of the sample surface in the work by Kuroda et al. ${ }^{[54]}$ (d) Scheme of the pyramidal QD structure. ${ }^{[52]}$ (e) Profile of the pyramidal InGaAs1-xNx QDs (the upper figure is the atomic force microscope results, the lower figure shows the SEM result of the sample after removing the substrate) ${ }^{[53]}$ Figures are adapted from Refs. 50, 52-54.

GaAs/aluminum gallium arsenide (AlGaAs) QDs are an interesting alternative to the conventional InAs/GaAs QDs. ${ }^{[49,55]}$ In 2013, Kuroda et al. used a droplet epitaxy method to realize self-assembled GaAs QDs on (111)A substrates $^{[54]}$ (see Fig. 10(c)). These QDs are highly symmetric in their shape. It was demonstrated that the 5\% of QDs show no detectable FSS and an entanglement fidelity of up to 0.86. In 2004, the same group also reported on the growth of InAs/indium aluminum arsenide (InAlAs) QDs on a $\mathrm{C}_{3 \mathrm{v}} \mathrm{InP}$ (111)A plane, with a vanishingsmall FSS and emissions in the telecom band. ${ }^{[56]}$

Another emerging possibility to obtain highly-symmetric growths of GaAs/AlGaAs QDs is to use the droplet etching and nanohole etching techniques. ${ }^{[57-60]}$ Recently, Huber et al. reported that, with preselected QDs in the sample, emissions of entangled photons can be obtained with high single-photon purity, high indistinguishability and high entanglement fidelity $(0.94 \pm 0.01) .{ }^{[61]}$ In the meanwhile, Keil and Zopf et al. realized a large ensemble of QD-based PEPS on a wafer without any post-growth tuning. ${ }^{[62]}$ Thanks to the growth optimization, the QDs on the whole wafer present an average FSS of $4.8 \pm 2.4 \mu \mathrm{eV}$. Under two-photon excitations, approximately $100 \%$ of QDs can emit entangled photons with fidelity greater than 0.5 ; in this case, the QDs have an exciton radiative lifetime shorter than 220 ps. Moreover, a significant fraction of QDs is expected to exhibit a high fidelity (greater than 0.8 ) without any post-growth tuning, ${ }^{[62]}$ (see Fig. 11).

(a)

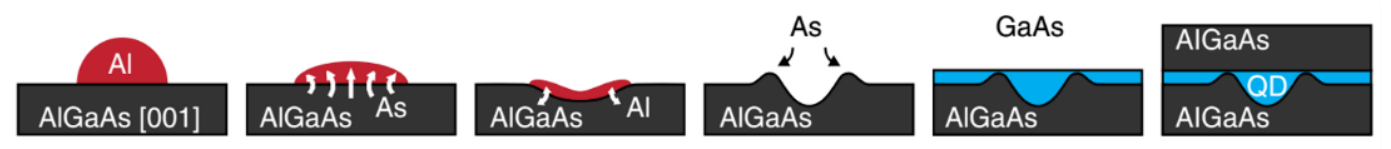

(b)

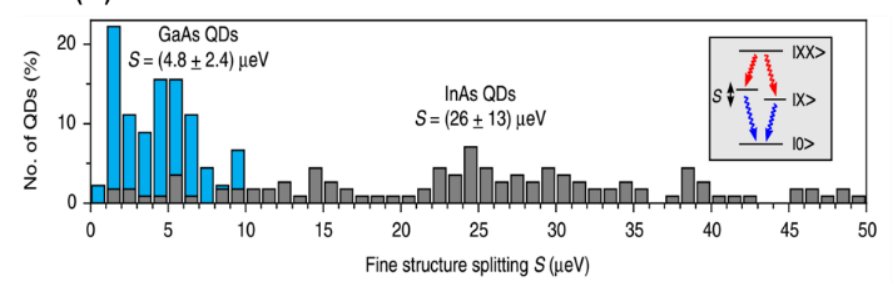

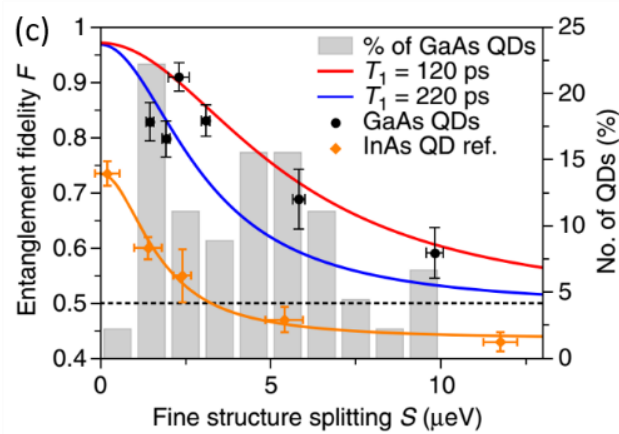

Figure 11. (a) Droplet etching and nanohole infilling technique for the GaAs QDs growth. (b) Comparison of FSS between two kinds of QDs. (c) Entanglement fidelity as a function of FSS. ${ }^{[62]}$ Figures are adapted from Ref. 62. 


\section{Eliminating FSS by post-growth methods}

Post-growth tuning knobs, such as the electric field, ${ }^{[63-67]}$ magnetic field, ${ }^{[68-70]}$ strain field, ${ }^{[47,}{ }^{71-85]}$ thermal annealing, ${ }^{[86-89]}$ and their combinations, can effectively manipulate the FSS of QDs. For practical applications of QD-based sources, these techniques are indispensable.

\section{(a) Electric field and magnetic field tuning}

The first breakthrough in electric field-controlled PEPS based on InAs QDs was reported by Bennett et al. in 2010. ${ }^{[67]}$ As shown in Fig. 12, the double AlGaAs high barriers prevent carriers from tunneling out the QDs when applying large electric fields, hence providing a large tuning scale of FSS. The variation of the exciton energy $\mathrm{F}$ with the external electric field can be described by $\Delta E_{H(\mathrm{~V})}=-p_{H(\mathrm{~V})} F+\beta F^{2}$, where $p_{H(\mathrm{~V})}$ is the permanent dipole moment in the $z$ direction of the exciton states with $\mathrm{H}$ and $\mathrm{V}$ polarized emissions, respectively. The result is shown in Fig. 12(c).

A similar work based on GaAs QDs was reported by Ghali et al. ${ }^{[63]}$ An n-i-Schottky diode was used to realize the electric field tuning of the QDs' FSS. The device structure and energy band profile along the growth direction are shown in Fig. 13. It can be observed that the FSS shows a nonlinear behavior with respect to the applied electric field, unlike the linear behavior of the InAs QDs reported in Ref. 67. The authors attributed this difference to the weak lateral confinement in the island-like GaAs QDs. Moreover, they obtained an entanglement fidelity of up to 0.72 .

Another interesting result was reported by Muller et al ${ }^{[90]} \mathrm{A}$ continuous-wave laser was used to tune the QDs in the alternating current Stark limit to eliminate the FSS. It was observed that FSS decreases as the intensity of the tuning laser increases.
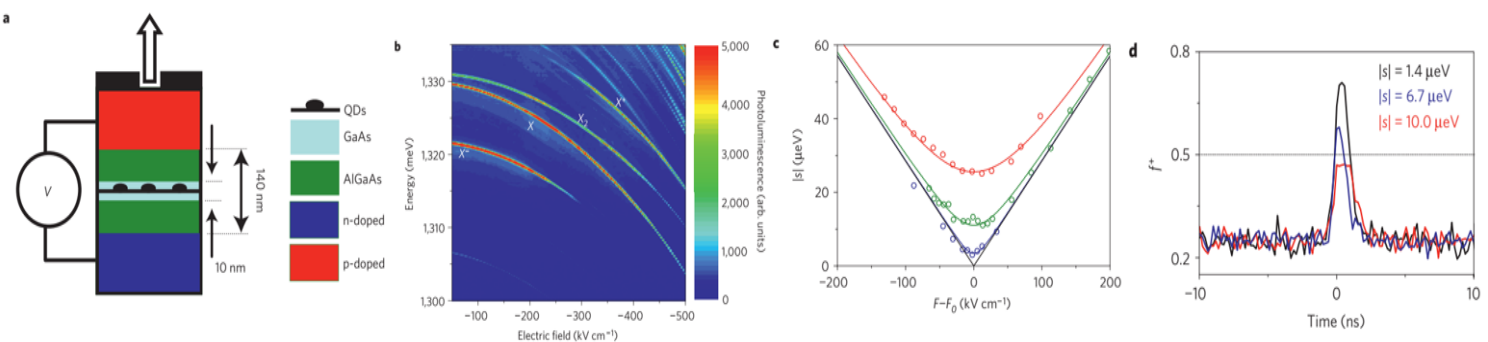

Figure 12. (a) and (b) Device design and observed giant Stark shift of the excitonic transitions. (c) FSS of three QDs controlled by vertical electric field. (d) Entanglement fidelity of three QDs with different FSS. ${ }^{[67]}$ Figures are adapted from Ref. 67.
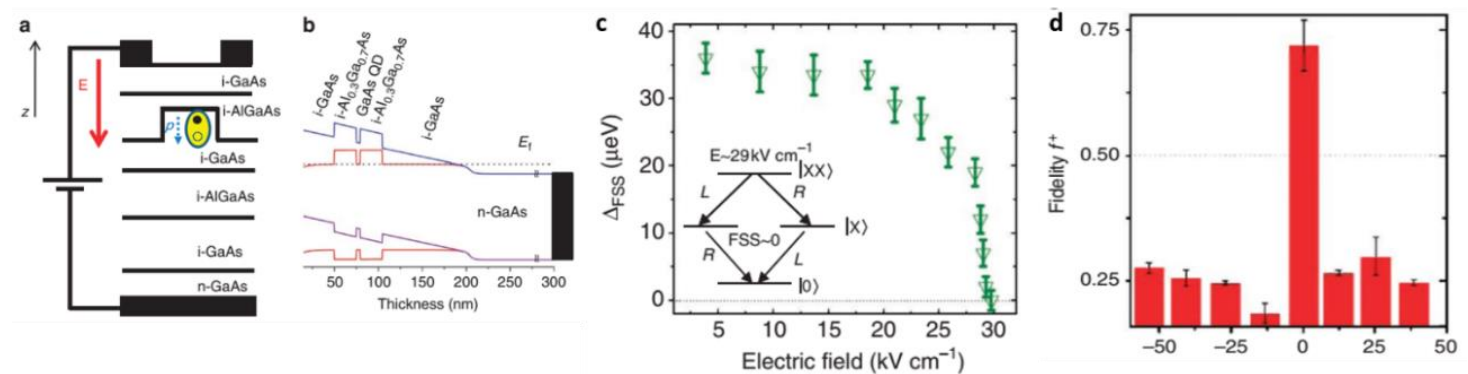

Figure 13. (a) Schottky-type device containing QDs. The polarization of QD exciton dipole moment is indicated by the dotted arrow. (b) z-direction energy band diagram. (c) Nonlinear dependence of FSS on external electric field. (d) Results of the entanglement fidelity. ${ }^{[63]}$ Figures are adapted from Ref. 63.

The effect of magnetic fields on QDs' FSS was studied in detail by Bayer et al. ${ }^{[0]}$ The first PEPS based on magnetic field tuning was reported by Stevenson et al. in 2006. ${ }^{[68]}$ Four years later, they reported that the inplane magnetic field can be used to tune the FSS more effectively, with a tuning coefficient of up to $1.05 \mu \mathrm{eV} \mathrm{T}^{-2}$. In 2014, Pooley et al. reported the elimination of FSS by applying vertical electric and in-plane magnetic fields at the same time. ${ }^{[91]}$ They reported that the FSS can be reduced to its minimum value and the emission energy can be tuned over several meVs with a 5-T magnet. 


\section{(b) FSS control by strain field}

The electrical field tuning may introduce excessive carriers around the QDs and degrade the coherence of the emitting photons. Furthermore, large electrical fields can lead to quenching effects. Nevertheless, the magnetic field tuning is not suitable for practical applications due to the bulky setup. An emerging technique consists in the usage of strain fields. Early works in this context were reported by Seidl et al..$^{[92]}$ and Ding et al. ${ }^{[71]}$ In particular, they used piezoelectric lead zirconic titanate $(\mathrm{PZT})$ and $\left[\mathrm{Pb}\left(\mathrm{Mg}_{1 / 3} \mathrm{Nb}_{2 / 3}\right) \mathrm{O}_{3}\right]_{0.72}\left[\mathrm{PbTiO}_{3}\right] 0.28(\mathrm{PMN}-\mathrm{PT})$, respectively, to tune the exciton states of the self-assembled QDs. In the latter work, thin membranes containing QDs were used to significantly increase the strain tuning range. Plumhof et al. reported the influences of anisotropic strain fields on the FSS of InAs and GaAs QDs, for the first time. ${ }^{[72]}$ Trotta $e t$ al. realized the simultaneous application of large strain and electric fields, leading to the successful elimination of FSS for almost each QDs in the sample. ${ }^{[75]}$ In 2012, a strain-tunable quantum light-emitting diode (QLED) was also reported..$^{[74]}$

The first experimental demonstration of strain-tunable QLED emitting polarization-entangled photon pairs (also called ELED) was realized by Zhang et al. ${ }^{[79]}$ As shown in Fig. 14(a), an n-i-p structure, with embedded InGaAs QDs, was integrated into a PMN-PT substrate. The anisotropic in-plane strain fields provided by the PMN-PT can be used to effectively manipulate the FSS (see Fig. 14(c)). Figure 14(d) shows the real part of the density matrix of the two-photon state. The entanglement fidelity as a function of FSS is shown in Fig. 14(b). The ELED can emit entangled photons with a repetition rate of up to $400 \mathrm{MHz}$. Recently, there has been also one report on the strain-tunable single photon QLED based on GaAs/AlGaAs QDs. ${ }^{[85]}$

a
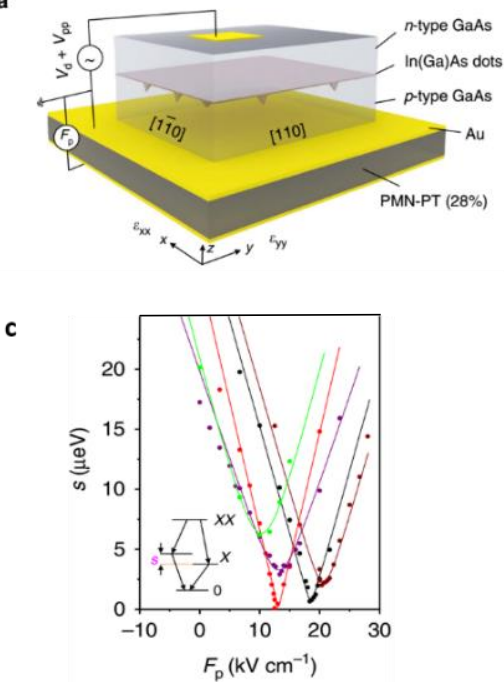
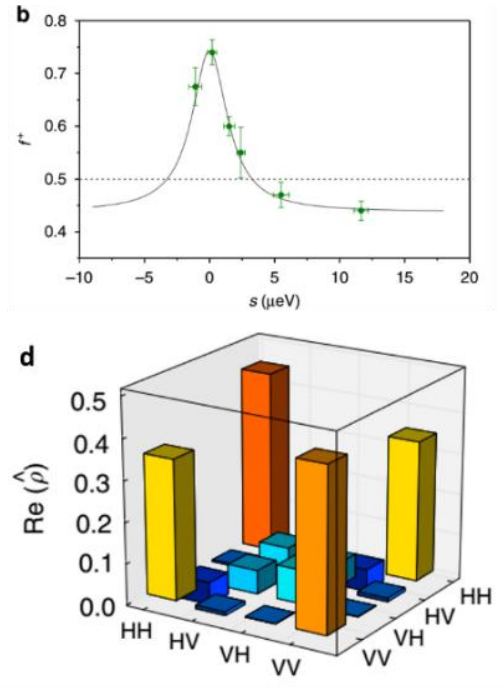

Figure 14. (a) Sketch of the diode structure. The PMN-PT was cut in a way that it can exert large anisotropic strain fields on the bonded ELED. (b) Entanglement fidelity as a function of FSS. (c) Representative variation of FSS of the high-energy component of the exciton as a function of the strain tuning for five QDs. (d) Real part of the density matrix of the two-photon state. ${ }^{[79]}$ Figures are adapted from Ref. 79.

For most of the tuning techniques, it is impossible to realize the simultaneous tuning of FSS and the QD emission wavelength. As a consequence, it is difficult to realize an entanglement swapping between two separated QDs. In 2015, an in-plane strain tuning stage with three degrees of freedom, where the FSS and the exciton energy can be independently tuned, was theoretically investigated ${ }^{[82]}$ The proposed device is shown in Fig. 15(a). Three pairs of piezoelectric strain arms are separated by 60 degrees. During the manipulation, the strains are controlled in the three different directions by the voltages $V_{1}, V_{2}$ and $V_{3}$, respectively. Another theoretical work by Wang et al. proposed that a three-dimensional stressor can be used for the same purpose (see Fig. 15(b)). ${ }^{[81]}$ The in-plane strain fields controlled by $V_{y}$ and $V_{z}$ are used to eliminate the FSS, while another PZT on top of the QDs is used to manipulate the exciton energy. 
(a)

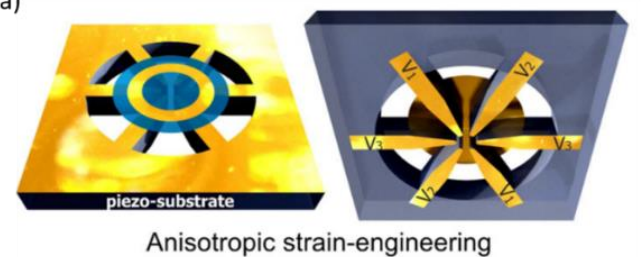

(b)

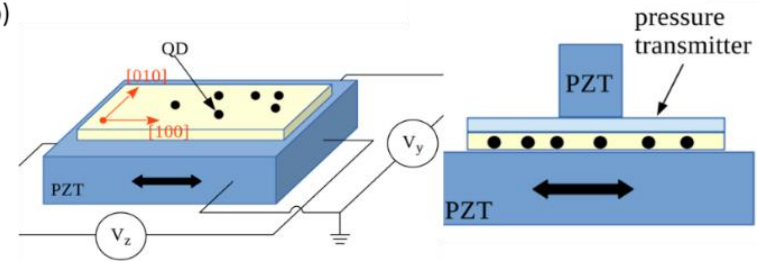

Figure 15. (a) Sketch of the strains stage providing anisotropic in-plane strain fields. Three independent voltages $\left(\mathrm{V}_{1,2,3}\right)$ are applied across the three pairs of legs. ${ }^{[82]}$ Figures are adapted from Ref. 82. (b) Sketch of a three-dimensional strain stage to independently tune the FSS and exciton energy in QDs. ${ }^{[81]}$ Figures are adapted from Ref. 81.

In 2016, a six-legged piezoelectric device was realized experimentally by Trotta et al. ${ }^{[80]}$ Polarization-entangled photons from InGaAs QDs were interfaced with a Cs vapor cell. After slowing down the photons by the atoms, a decrease in the entanglement fidelity was observed. This work opens the door to the realization of a hybrid quantum system based on semiconductor QDs. The device structure and entanglement fidelity are shown in Figs. 16(a) and 16(b), respectively. In 2016, Chen et al. also realized an on-chip integrated wavelength tunable PEPS based on InGaAs QDs. The unique advantages of their device are the ultra-small footprint and ultra-low tuning voltage, which are important for the fabrication of advanced quantum photonic circuits for on-chip QIP applications.

a

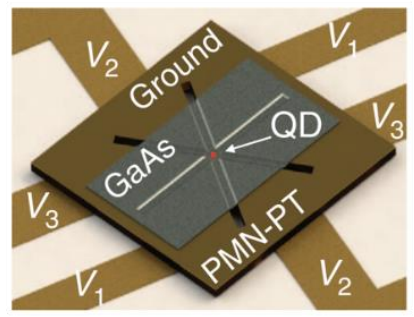

C

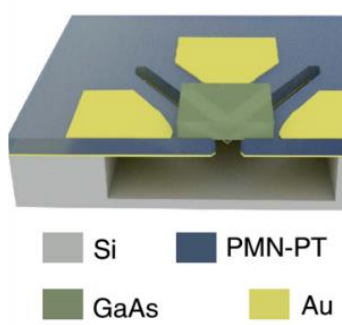

b

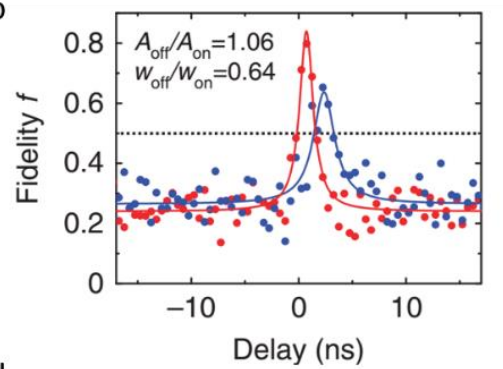

d

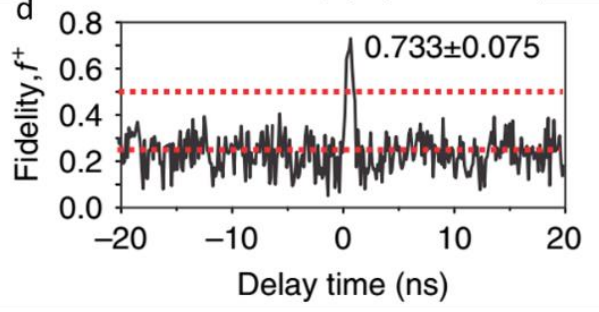

Figure 16. (a) Sketch of the six-legged strain tuning device used to engineer a nanomembrane (grey region) containing QDs. (b) Entanglement fidelity with (blue data points) and without (red data points) the Cs cell in the exciton optical path. ${ }^{[80]}$ Figures are adapted from Ref. 80. (c) Scheme of the cross section of the four-legged strain-tuning device. A focused ion beam (FIB) and a wet-chemical undercut were used to fabricate the device. A thin GaAs nanomembrane containing In(Ga)As QDs was transferred to the suspended region between the four legs. ${ }^{[83]}$ (d) Entanglement fidelity results after the FSS tuning, without any background subtraction. Figures are adapted from Ref. 83.

\section{Brightness enhancement by photonic structures}

To date, one of the most challenging tasks for QD-based PEPS is to enhance their brightness. Due to the high refractive index of the surrounding materials and isotropic emission of QDs, only a small portion of the photon pairs can be collected. The most common solution adopted to improve the extraction efficiency is to use the Purcell effect. When the QDs are coupled both spatially and spectrally with a cavity mode, their photon emissions are modified by 


$$
\frac{\Gamma_{c a v}}{\Gamma_{\text {free }}}=\frac{3 Q \lambda^{3}}{4 \pi^{2} V} .
$$

Where $\mathrm{Q}$ and $\lambda$ are the quality factor and resonant wavelength of the cavity, respectively, and $\mathrm{V}$ is the mode volume. The high-Q cavity scheme has been widely exploited to enhance the brightness of single-photon sources. However, due to the apparent energy difference between the exciton and biexciton photons, it is difficult to translate this scheme to QD-based PEPS.

An elegant solution was proposed by Dousse et al. ${ }^{[93]}$ As shown in Fig. 17(a), two GaAs/AlGaAs micropillars, with $\mathrm{Q}$ factors equal to 4000 and 60000 , respectively, are used to enhance the extraction efficiency of entangled photons from a single InAs QD. By precisely choosing the pillar diameters and distance, the exciton and biexciton photons can be coupled to the two cavity modes, respectively (see Fig. 17(c)). They reached a pair rate of 0.12 per excitation pulse into the first lens.

An alternative option, which also brings a broadband enhancement, is to use the nanowire QDs. Versteegh et $a l .{ }^{[94]}$ reported that the nanowire waveguide has the advantages of a broad frequency bandwidth and a controlled direction, which suits for the QD-based PEPS. The light extraction efficiency of a single InAsP QD in InP nanowire could reach $18 \pm 3 \%$. Besides, the entanglement fidelity to the maximum Bell state can be up to 0.765 , without any time-gating technique. A similar work was reported by Huber $e t$ al. ${ }^{[55]}$ The device structure is shown in Fig. 17(d). It is worth noting that the nanowire QDs can also be strain tunable, as reported by Chen et al. ${ }^{[96]}$
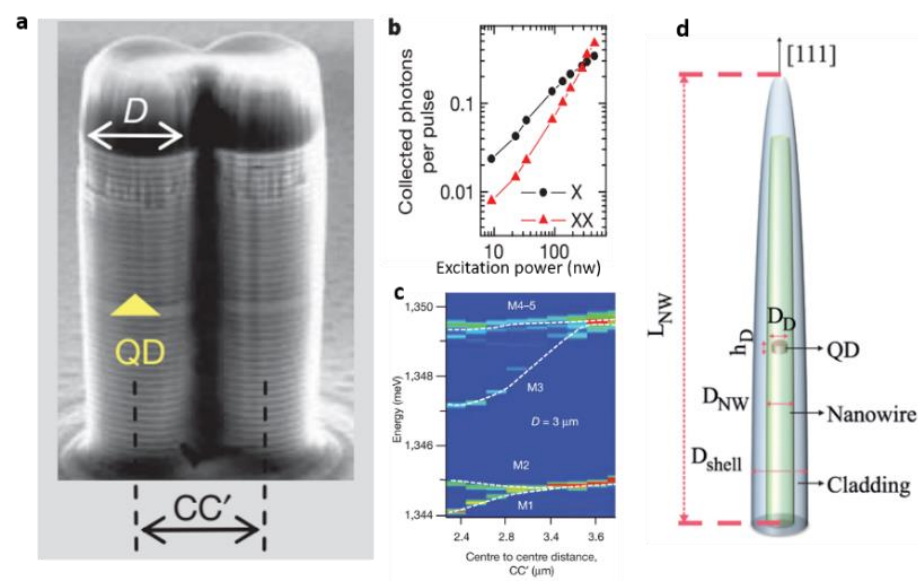

Figure 17. (a) Diagram of the device that contains two microcavities and one InAs QD. (b) Number of exciton and biexciton photons collected in each excitation pulse as a function of the excitation power. (c) The optical modes of the photonic molecule as a function of the intra-molecule distance. ${ }^{[93]}$ Figures are adapted from Ref. 93. (d) Structure of nanowire QDs. ${ }^{[95]}$ The figure is adapted from Ref. 95.

\section{Optimizing the scheme of excitations}

Semiconductor QDs can be excited either optically or electrically. The electrical injection is more promising in practical QIP, given that no sophisticated laser systems are required. The first ELED was realized by Salter $e t$ $a l .{ }^{[97]}$ They showed that the device could emit polarization-entangled photon pairs under DC or AC injections, with the latter achieving an entanglement fidelity of up to 0.82 . After this pioneering work, it took the community a long time to reproduce the result, simply due to the low probability of finding QDs with small enough FSS. Zhang et al. reported a strain-tunable ELED, ${ }^{[79]}$ as above mentioned. Another interesting recent work from Chung et al. ${ }^{[98]}$ is based on the realization of selectively carrier-injected and site-controlled arrays of ELED based on pyramidal QDs.

For optical excitations, QDs can be excited either resonantly or non-resonantly. For the non-resonant excitation, the carriers are excited to energy levels higher than the first excited states, and they decay to the first excited states of QDs. In this scheme, an inevitable time jitter of the emissions is created. Besides, charges in excess will form around the QDs, leading to spectral diffusions and a pure dephasing. The combination of these effects will significantly degrade both the indistinguishability and coherence of the QD emissions. Moreover, the biexciton population probability under the non-resonant excitation is quite low. In 2014, Müller et al. realized a twophoton excitation (TPE) to single QDs via a virtual state. ${ }^{[99]}$ The schematic is shown in Fig. 18(a). 

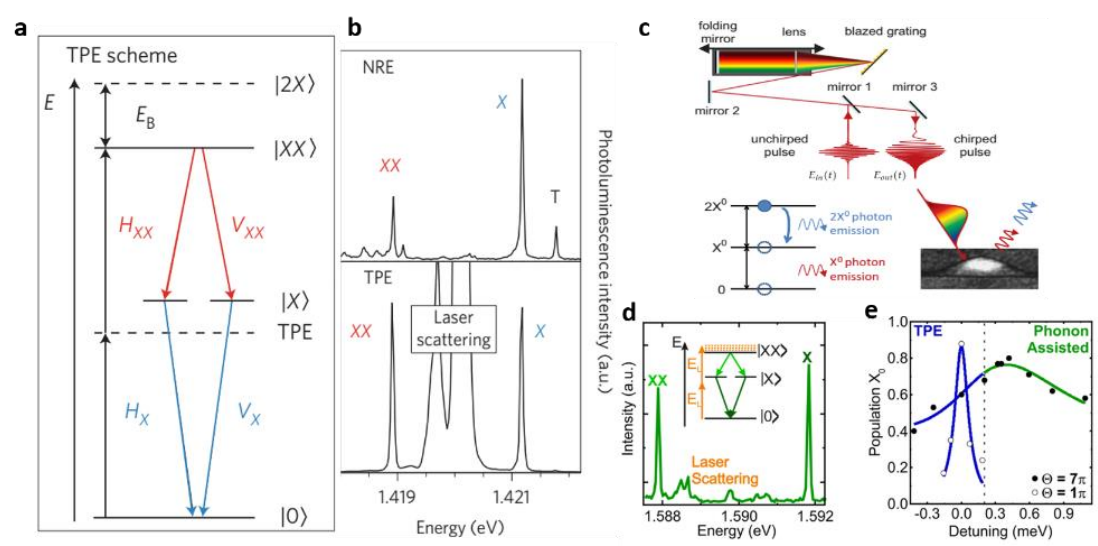

Figure 18. (a) Energy diagram of TPE. (b) Photofluorescence spectra of a same QD, under non-resonant excitation (upper) and TPE (lower). ${ }^{[99]}$ Figures are adapted from Ref. 99. (c) Scheme of a pulse shaper to generate the chirp pulse (upper), and generation of a biexciton with broadband, chirped excitation (lower). ${ }^{[100]}$ Figures are adapted from Ref. 100. (d) Photofluorescence spectrum of a GaAs QD under phonon-assisted TPE for optimized detuning of the laser energy, pulse length $\left(\mathrm{EL}_{\mathrm{L}}=1.5901 \mathrm{eV}, \tau_{\mathrm{p}}=10 \mathrm{ps}\right)$ and a pulse area of $6 \pi$. (e) Population of the exciton state as a function of the laser detuning for varying the excitation power. ${ }^{[101]}$ Figures are adapted from Ref. 101.

Figure 18(b) shows the photoluminescence spectra of a same QD, under either non-resonant excitation or TPE. Under TPE, the biexciton almost has the same intensity as that of the exciton. It is worth noting that the generation efficiency of the biexciton increases by a factor of 4 under resonant excitations. ${ }^{[99]}$ Their source also shows high entanglement fidelity and indistinguishability. Similar works were reported by several other groups. ${ }^{[61,62]}$

However, TPE is sensitive to the excitation power and the QDs' environment, and a slight perturbation may lead to dramatic variations in the population probability. Reindl et al. reported a scheme called phonon-assisted TPE. ${ }^{[101]}$ They claimed that, although the phonon-assisted TPE scheme is inherently non-resonant, the population inversion of exciton and biexciton states coupled to a quasi-continuum of vibrational modes is possible (see the inset of Fig. 18(d)). As a result, the population is robust against the detuning of the laser energy compared to traditional TPE, as shown in Fig. 18(e). Another alternative scheme is the adiabatic rapid passage (ARP), which is a resonant excitation technique. In the ARP, a broadband and chirped pulse is used to excite the QDs. According to several reports, ${ }^{[100,102]}$ the biexciton occupation is robust against the excitation power of the positive chirp. The experimental setup and excitation illustration are shown in Fig. 18(c).

\section{Outlook}

Since the first realization of a QD-based entangled photon source in 2006, this field has experienced a significant success. The "on-demand" emissions of QDs make them a strong competitor of the traditional SPDC-based sources. To date, the FSS has been controlled through the methods mentioned above. There are also progresses in the on-chip integration, development of efficient excitation schemes, improvement of the photon indistinguishability, etc. However, several challenges remain to be overcome. For example, it is necessary to explore new material systems that function at higher or even room temperatures. In addition, the low brightness is a main hurdle. Recently, micro-lens fabricated with in-situ electron-beam lithography ${ }^{[103]}$ and micro-objective made with a 3D-printing technique ${ }^{[104]}$ have been used in QD-based single photon source. These interesting techniques can be exploited for the fabrication of QD-based PEPS. An efficient photon extraction from a QD may be also achieved with a broadband planar cavity antenna. ${ }^{[105]}$

For the application of PEPS, the entanglement swapping and purification protocols are important for an efficient distribution of the entanglement in a network. ${ }^{[106]}$ Such protocols have been experimentally realized with SPDC sources. ${ }^{[107,108]}$ Recently, the quantum secure direct communication has also been experimentally realized. ${ }^{[109,110]}$ Thanks to the fast progress of QDs, we believe that QD-based PEPS will soon become an important workhorse for advanced quantum optics experiments.

\section{References}

1. Einstein A, Podolsky B, and Rosen N 1935 Physical Review 47777

2. Raussendorf R, Browne D E, and Briegel H J 2003 Physical Review A 68022312

3. Knill E 2010 Nature 463441 
4. Gisin N, Ribordy G, Tittel W, and Zbinden H 2002 Reviews of Modern Physics 74145

5. Ekert A K 1991 Physical review letters 67661

6. Bouwmeester D, Pan J-W, Mattle K, Eibl M, Weinfurter H, and Zeilinger A 1997 Nature 390 575

7. Furusawa A, Sørensen J L, Braunstein S L, Fuchs C A, Kimble H J, and Polzik E S 1998 Science 282706

8. Blatt R, and Wineland D 2008 Nature 4531008

9. Langer C, Ozeri R, Jost J D, Chiaverini J, Demarco B, Ben-Kish A, Blakestad R B, Britton J, Hume D B, Itano W M, Leibfried D, Reichle R, Rosenband T, Schaetz T, Schmidt P O, and Wineland D J 2005 Physical review letters 95060502

10. Roch N, Schwartz M E, Motzoi F, Macklin C, Vijay R, Eddins A W, Korotkov A N, Whaley K B, Sarovar M, and Siddiqi I 2014 Physical review letters 112170501

11. Oliver W D, Yamaguchi F, and Yamamoto Y 2002 Phys Rev Lett 88037901

12. Wang C, Zhang Y, and Jin G-s 2011 Physical Review A 84032307

13. Lee K C, Sprague M R, Sussman B J, Nunn J, Langford N K, Jin X-M, Champion T, Michelberger P, Reim K F, and England D 2011 Science 3341253

14. Gao W B, Fallahi P, Togan E, Miguel-Sanchez J, and Imamoglu A 2012 Nature 491426

15. Pan J W, Daniell M, Gasparoni S, Weihs G, and Zeilinger A 2001 Physical review letters 86 4435

16. Fox M 2006 Quantum optics: an introduction (Oxford: OUP Oxford) p. 268

17. Michler P 2017 Quantum Dots for Quantum Information Technologies (Springer) p. 237

18. Wang J, Yang J-Y, Fazal I M, Ahmed N, Yan Y, Huang H, Ren Y, Yue Y, Dolinar S, Tur M, and Willner A E 2012 Nature Photonics 6488

19. Allen L, Beijersbergen M W, Spreeuw R J C, and Woerdman J P 1992 Physical Review A 45 8185

20. Mirhosseini M, Magaña-Loaiza O S, O'Sullivan M N, Rodenburg B, Malik M, Lavery M P J, Padgett M J, Gauthier D J, and Boyd R W 2015 New Journal of Physics 17033033

21. Nagali E, Sciarrino F, De Martini F, Marrucci L, Piccirillo B, Karimi E, and Santamato E 2009 Physical review letters 103013601

22. Wang X L, Cai X D, Su Z E, Chen M C, Wu D, Li L, Liu N L, Lu C Y, and Pan J W 2015 Nature 518 516

23. Brendel J, Gisin N, Tittel W, and Zbinden H 1999 Physical review letters 822594

24. Orieux A, Versteegh M A M, Jons K D, and Ducci S 2017 Reports on progress in physics. Physical Society 80076001

25. Aspect A, Grangier P, and Roger G 1981 Physical review letters 47460

26. Aspect $A$, Grangier $P$, and Roger $G 1982$ Physical review letters 4991

27. Edamatsu K 2007 Japanese Journal of Applied Physics 467175

28. Ling A, Lamas-Linares A, and Kurtsiefer C 2008 Physical Review A 77043834

29. Michler $P 2009$ Single semiconductor quantum dots (Springer) p. 231

30. Benson O, Santori C, Pelton M, and Yamamoto Y 2000 Physical Review Letters 842513

31. Rosenfeld W, Burchardt D, Garthoff R, Redeker K, Ortegel N, Rau M, and Weinfurter H 2017 Physical review letters 119010402

32. Handsteiner J, Friedman A S, Rauch D et al. 2017 Physical review letters 118060401 
33. Christensen B G, McCusker K T, Altepeter J B, Calkins B, Gerrits T, Lita A E, Miller A, Shalm L K, Zhang Y, Nam S W, Brunner N, Lim C C, Gisin N, and Kwiat P G 2013 Physical review letters 111 130406

34. Giustina M, Mech A, Ramelow S, Wittmann B, Kofler J, Beyer J, Lita A, Calkins B, Gerrits T, Nam S W, Ursin R, and Zeilinger A 2013 Nature 497227

35. Pan J-W, Bouwmeester D, Daniell M, Weinfurter H, and Zeilinger A 2000 Nature 403515

36. Freedman S J, and Clauser J F 1972 Physical review letters 28938

37. Tittel W, Brendel J, Zbinden H, and Gisin N 1998 Physical review letters 813563

38. James D F V, Kwiat P G, Munro W J, and White A G 2001 Physical Review A 64030302

39. Clauser J F, Horne M A, Shimony A, and Holt R A 1969 Physical Review Letters 23880

40. Altepeter J B, Jeffrey E R, and Kwiat P G 2005 Advances in Atomic, Molecular, and Optical Physics $\mathbf{5 2} 105$

41. Lodahl P, Mahmoodian S, and Stobbe S 2015 Reviews of Modern Physics 87347

42. Stevenson R M, Hudson A J, Bennett A J, Young R J, Nicoll C A, Ritchie D A, and Shields A J 2008 Phys Rev Lett 101170501

43. Bester G, Nair S, and Zunger A 2003 Physical Review B 67161306

44. Bester $\mathrm{G}$, and Zunger A 2005 Physical Review B 71045318

45. Narvaez G A, Bester G, and Zunger A 2005 Physical Review B 72245318

46. Singh R, and Bester G 2010 Phys Rev Lett 104196803

47. Gong M, Zhang W, Guo G C, and He L 2011 Phys Rev Lett 106227401

48. Seguin R, Schliwa A, Rodt S, Potschke K, Pohl U W, and Bimberg D 2005 Phys Rev Lett 95 257402

49. Rastelli A, Stufler S, Schliwa A, Songmuang R, Manzano C, Costantini G, Kern K, Zrenner A, Bimberg D, and Schmidt O G 2004 Physical review letters 92166104

50. Schliwa A, Winkelnkemper M, Lochmann A, Stock E, and Bimberg D 2009 Physical Review B 80161307

51. Singh R, and Bester G 2009 Phys Rev Lett 103063601

52. Mohan A, Felici M, Gallo P, Dwir B, Rudra A, Faist J, and Kapon E 2010 Nature Photonics 4302

53. Juska G, Dimastrodonato V, Mereni L O, Gocalinska A, and Pelucchi E 2013 Nat Photon 7527

54. Kuroda T, Mano T, Ha N, Nakajima H, Kumano H, Urbaszek B, Jo M, Abbarchi M, Sakuma Y, Sakoda K, Suemune I, Marie X, and Amand T 2013 Physical Review B 88041306

55. Chekhovich E A, Makhonin M N, Tartakovskii A I, Yacoby A, Bluhm H, Nowack K C, and Vandersypen L M 2013 Nature materials 12494

56. Liu X, Ha N, Nakajima H, Mano T, Kuroda T, Urbaszek B, Kumano H, Suemune I, Sakuma Y, and Sakoda K 2014 Physical Review B 90081301

57. Wang Z M, Liang B L, Sablon K A, and Salamo G J 2007 Applied Physics Letters 90113120

58. Stemmann A, Heyn C, Köppen T, Kipp T, and Hansen W 2008 Applied Physics Letters 93 123108

59. Heyn C, Stemmann A, Köppen T, Strelow C, Kipp T, Grave M, Mendach S, and Hansen W 2009 Applied Physics Letters 94183113

60. Huo Y H, Rastelli A, and Schmidt O G 2013 Applied Physics Letters 102152105

61. Huber D, Reindl M, Huo Y, Huang H, Wildmann J S, Schmidt O G, Rastelli A, and Trotta R 2017 Nat Commun 815506

62. Keil R, Zopf M, Chen Y, Hofer B, Zhang J, Ding F, and Schmidt O G 2017 Nat Commun 815501 
63. Ghali M, Ohtani K, Ohno Y, and Ohno H 2012 Nat Commun 3661

64. Gerardot B D, Seidl S, Dalgarno P A, Warburton R J, Granados D, Garcia J M, Kowalik K, Krebs O, Karrai K, Badolato A, and Petroff P M 2007 Applied Physics Letters 90041101

65. Mar J D, Baumberg J J, Xu X L, Irvine A C, and Williams D A 2016 Physical Review B 93045316

66. Vogel M M, Ulrich S M, Hafenbrak R, Michler P, Wang L, Rastelli A, and Schmidt O G 2007 Applied Physics Letters 91051904

67. Bennett A J, Pooley M A, Stevenson R M, Ward M B, Patel R B, de la Giroday A B, Skold N, Farrer I, Nicoll C A, Ritchie D A, and Shields A J 2010 Nat Phys 6947

68. Stevenson R M, Young R J, Atkinson P, Cooper K, Ritchie D A, and Shields A J 2006 Nature 439 179

69. Mrowiński P, Musiał A, Maryński A, Syperek M, Misiewicz J, Somers A, Reithmaier J P, Höfling S, and Sęk G 2015 Applied Physics Letters 106053114

70. Bayer M, Ortner G, Stern O, Kuther A, Gorbunov A A, Forchel A, Hawrylak P, Fafard S, Hinzer K, Reinecke T L, Walck S N, Reithmaier J P, Klopf F, and Schäfer F 2002 Physical Review B 65195315

71. Ding F, Singh R, Plumhof J D, Zander T, Krapek V, Chen Y H, Benyoucef M, Zwiller V, Dorr K, Bester G, Rastelli A, and Schmidt O G 2010 Phys Rev Lett 104067405

72. Plumhof J D, Křápek V, Ding F, Jöns K D, Hafenbrak R, Klenovský $P$, Herklotz A, Dörr K, Michler P, Rastelli A, and Schmidt O G 2011 Physical Review B 83121302

73. Rastelli A, Ding F, Plumhof J D et al. 2012 physica status solidi (b) 249687

74. Trotta R, Atkinson P, Plumhof J D, Zallo E, Rezaev R O, Kumar S, Baunack S, Schroter J R, Rastelli A, and Schmidt O G 2012 Advanced materials 242668

75. Trotta R, Zallo E, Ortix C, Atkinson P, Plumhof J D, van den Brink J, Rastelli A, and Schmidt O G 2012 Physical Review Letters 109147401

76. Plumhof J D, Trotta R, Křápek V, Zallo E, Atkinson P, Kumar S, Rastelli A, and Schmidt O G 2013 Physical Review B 87075311

77. Kumar S, Zallo E, Liao Y H, Lin P Y, Trotta R, Atkinson P, Plumhof J D, Ding F, Gerardot B D, Cheng S J, Rastelli A, and Schmidt O G 2014 Physical Review B 89115309

78. Trotta R, Wildmann J S, Zallo E, Schmidt O G, and Rastelli A 2014 Nano letters 143439

79. Zhang J, Wildmann J S, Ding F, Trotta R, Huo Y, Zallo E, Huber D, Rastelli A, and Schmidt O G 2015 Nat Commun 610067

80. Trotta R, Martin-Sanchez J, Wildmann J S, Piredda G, Reindl M, Schimpf C, Zallo E, Stroj S, Edlinger J, and Rastelli A 2016 Nat Commun 710375

81. Wang J, Gong M, Guo G C, and He L 2015 Phys Rev Lett 115067401

82. Trotta R, Martin-Sanchez J, Daruka I, Ortix C, and Rastelli A 2015 Phys Rev Lett 114150502

83. Chen Y, Zhang J, Zopf M, Jung K, Zhang Y, Keil R, Ding F, and Schmidt O G 2016 Nat Commun 7 10387

84. Höfer B, Zhang J, Wildmann J, Zallo E, Trotta R, Ding F, Rastelli A, and Schmidt O G 2017 Applied Physics Letters 110151102

85. Huang H, Trotta R, Huo Y, Lettner T, Wildmann J S, Martin-Sanchez J, Huber D, Reindl M, Zhang J, Zallo E, Schmidt O G, and Rastelli A 2017 ACS photonics 4868

86. Langbein W, Borri P, Woggon U, Stavarache V, Reuter D, and Wieck A D 2004 Physical Review B 69161301

87. Tartakovskii A I, Makhonin M N, Sellers I R, Cahill J, Andreev A D, Whittaker D M, Wells J P R, Fox A M, Mowbray D J, Skolnick M S, Groom K M, Steer M J, Liu H Y, and Hopkinson M 2004 Physical Review B 70193303 
88. Ellis D J P, Stevenson R M, Young R J, Shields A J, Atkinson P, and Ritchie D A 2007 Applied Physics Letters 90011907

89. Seguin R, Schliwa A, Germann T D, Rodt S, Pötschke K, Strittmatter A, Pohl U W, Bimberg D, Winkelnkemper M, Hammerschmidt T, and Kratzer P 2006 Applied Physics Letters 89263109

90. Muller A, Fang W, Lawall J, and Solomon G S 2009 Phys Rev Lett 103217402

91. Pooley M A, Bennett A J, Stevenson R M, Shields A J, Farrer I, and Ritchie D A 2014 Physical Review Applied 1024002

92. Seidl S, Kroner M, Högele A, Karrai K, Warburton R J, Badolato A, and Petroff P M 2006 Applied Physics Letters 88203113

93. Dousse A, Suffczynski J, Beveratos A, Krebs O, Lemaitre A, Sagnes I, Bloch J, Voisin P, and Senellart P 2010 Nature 466217

94. Versteegh M A, Reimer M E, Jons K D, Dalacu D, Poole P J, Gulinatti A, Giudice A, and Zwiller V 2014 Nat Commun 55298

95. Huber T, Predojevic A, Khoshnegar M, Dalacu D, Poole P J, Majedi H, and Weihs G 2014 Nano letters 147107

96. Chen $Y$, Zadeh I E, Jöns K D, Fognini A, Reimer M E, Zhang J, Dalacu D, Poole P J, Ding F, Zwiller V, and Schmidt O G 2016 Applied Physics Letters 108182103

97. Salter C L, Stevenson R M, Farrer I, Nicoll C A, Ritchie D A, and Shields A J 2010 Nature 465 594

98. Chung T H, Juska G, Moroni S T, Pescaglini A, Gocalinska A, and Pelucchi E 2016 Nature Photonics 10782

99. MullerM, BounouarS, Jons K D, GlassIM, and MichlerP 2014 Nat Photon 8224

100. Kaldewey $T$, Lüker $S$, Kuhlmann A V, Valentin $S$ R, Ludwig A, Wieck A D, Reiter D E, Kuhn T, and Warburton R J 2017 Physical Review B 95161302

101. Reindl M, Jons K D, Huber D, Schimpf C, Huo Y, Zwiller V, Rastelli A, and Trotta R 2017 Nano letters 174090

102. Wei Y-J, He Y-M, Chen M-C, Hu Y-N, He Y, Wu D, Schneider C, Kamp M, Höfling S, and Lu C-Y 2014 Nano letters 146515

103. Gschrey M, Thoma A, Schnauber P, Seifried M, Schmidt R, Wohlfeil B, Kruger L, Schulze J H, Heindel T, Burger S, Schmidt F, Strittmatter A, Rodt S, and Reitzenstein S 2015 Nat Commun 67662

104. Fischbach S, Schlehahn A, Thoma A, Srocka N, Gissibl T, Ristok S, Thiele S, Kaganskiy A, Strittmatter A, Heindel T, Rodt S, Herkommer A, Giessen H, and Reitzenstein S 2017 ACS photonics 4 1327

105. Chen X-W, Götzinger S, and Sandoghdar V 2011 Opt. Lett. 363545

106. Pan J-W, Chen Z-B, Lu C-Y, Weinfurter H, Zeilinger A, and Żukowski M 2012 Reviews of Modern Physics 84777

107. Pan J-W, Bouwmeester D, Weinfurter H, and Zeilinger A 1998 Physical review letters 803891

108. Pan J-W, Gasparoni S, Ursin R, Weihs G, and Zeilinger A 2003 Nature 423417

109. Zhang W, Ding D S, Sheng Y B, Zhou L, Shi B S, and Guo G C 2017 Phys Rev Lett 118220501

110. Zhu F, Zhang W, Sheng Y, and Huang Y 2017 Science Bulletin 621519 